\title{
DERECHO Y POLÍTICAS AMBIENTALES EN PORTUGAL (SEGUNDO SEMESTRE 2019)
}

\author{
AMPARO SERENO ROSADO \\ Investigadora Integrada del OBSERVARE \\ Universidade Autónoma de Lisboa (UAL)
}

Profesora en el "Instituto Superior de Contabilidade e Administração de Lisboa" (ISCAL) 
Sumario: 1. Introducción. 2. El Tajo, sus problemas ambientales y el Convenio de Albufeira. 3. El refuerzo de la política de combate a los incendios y ordenación forestal. 4. El enorme avanzo de la movilidad eléctrica empieza a dar claros frutos. 5. Participación Pública. 5.1. Nuevo aeropuerto de Lisboa. 5.2. Programa Nacional de la Política de Ordenación del Territorio. 6. Otras novedades de interés: neutralidad carbónica, economía circular y Lisboa Capital Verde 2020.

\section{INTRODUCCIÓN}

Este segundo semestre viene marcado por las novedades que el nuevo gobierno - salido de las últimas elecciones nacionales de 6 de octubre de 2019 - trae para el Ministerio del Ambiente. Este, que hace ahora un año, había salido reforzado con la cesión, por parte del Ministerio de Economía y Energía de sus competencias en materia energética - a partir de lo cual pasó a denominarse "Ministerio do Ambiente e da Transição Energética" (MATE) - no cambia de titular, pero cambia nuevamente de nomenclatura. Ahora se llama: "Ministério do Ambiente e da Ação Climática (MAAC)". No obstante, esta mudanza no es tan sólo nominal, sino que adquiere nuevas competencias - antes atribuidas al Ministerio de Agricultura - y en lo que se refiere a la ordenación forestal. Además, el próximo XXII Gobierno Constitucional - que el 26 de octubre pasado fue investido, divulgando, en ese mismo día, su Programa de Gobierno - promete una gran ambición en lo que se refiere a medidas ambientales, sobre todo a las relacionadas con la "descarbonización" de la economía. Por ejemplo, las dos únicas centrales eléctricas a carbón que funcionan en Portugal (Pego y Sines) y que ya el anterior Gobierno había anunciado que serían cerradas en 2023 y 2025, respectivamente, ahora se dice que lo serán, pero dos años antes (2021 y 2023). Esta promesa, que esperemos se cumpla, va a suponer un gran esfuerzo para un país que, como no tiene energía nuclear, tendrá, necesariamente que reforzar la hidroeléctrica, eólica y solar. Además, se promete una "revolución" en los transportes con el refuerzo en el ferrocarril, la manutención de los precios sociales de los transportes públicos en todo el territorio - estos fueron reducidos el año pasado para la mitad, (o más en algunos casos 1 ) y la continuación de la apuesta en la movilidad eléctrica.

\footnotetext{
1 Desde abril de 2019, en las áreas metropolitanas de Lisboa y de Oporto hay nuevas modalidades de pago mensual de los transportes públicos: uno que permitirá viajar por toda el área metropolitana (18 municipios, en el caso de Lisboa y 17 en Oporto) que costará 40 euros, lo que permitirá, en algunos casos, ahorros mensuales de más de 100 euros. Hasta ahora, el abono más caro de la Gran Lisboa costaba 192,15 euros,
} 
También se incluye en el Programa de Gobierno, el denominado "Pacto para la Europa Verde" que gobierno portugués pretendo negociar con los restantes socios europeos en el ámbito de la Presidencia portuguesa de la UE - en el primer semestre de 2021.

Por último, como "medida estrella" en materia de economía circular está la meta de abolir, hasta el final de 2020, los plásticos no reutilizables y la promesa, no menos difícil de cumplir, de renegociar con España el Convenio de Albufeira sobre cuencas internacionales, especialmente el Tajo, por sus graves problemas ambientales.

\section{EL TAJO, SUS PROBLEMAS AMBIENTALES Y EL CONVENIO DE} ALBUFEIRA

Cada vez que hay sequia - y las mismas suceden con más frecuencia y severidad en el Centro y Sur de la Península, o sea a partir de la cuenca del Tajo - salen a la luz las noticias sobre el cumplimiento (o no) de los caudales acordados en el Convenio de Albufeira (CA) de 1998. En este Convenio se establecen los caudales mínimos que España debe entregar a Portugal cada año - determinados trimestralmente, semanalmente y, en el caso del Guadiana, también diariamente. Sin embargo, hay un patrón de comportamiento que se repite cada que hay sequía: la contradicción entre la información oficial (de los Ministerios de Ambiente portugués y español) que afirman que España cumple con los caudales mínimos y las fuentes de la sociedad civil - tales como: asociaciones de defensa del medio ambiente, pescadores, clubes náuticos fluviales y municipios ribereños del Tajo - que afirman justamente lo contrario. Así sucedió durante la sequía de 2017 y volvió a suceder nuevamente en octubre de 2019.

El año pasado los problemas de esta cuenca estuvieron relacionados más con la calidad del agua, pero, indirectamente, también con la cantidad. El 2018 comenzó con alarmantes noticias sobre contaminación de las aguas del Tajo

correspondiente a un combinado entre ferrocarril y la empresa "Barraqueiro Transportes". Con el nuevo "Navegante Metropolitano", los usuarios de estos transportes tendrán, por lo menos, un ahorro mensual de 152,15 euros. Cf. <https://www.publico.pt/2019/04/01/economia/noticia/vai-mudar-transportes-publicospartir-hoje-1867302>. 
proveniente de fuentes situadas en territorio portugués2. Para evitar nuevos accidentes, la "Agencia Portuguesa del Ambiente" (APA), finalmente, procedió a la revisión de las licencias de vertidos de aguas residuales3. Pero además de la "contaminación nacional", diversas ONGA portuguesas y asociaciones de ciudadanos se quejaban del mal estado de calidad de las aguas del Tajo provenientes de España4. Los distintos medios de comunicación portugueses se han hecho eco, durante 2019, de todas estas críticas, con titulares dramáticos como, por ejemplo: "Como Espanha envenena a água do Tejo 5". En este reportaje se realizó una descripción de los 1007 Km de recorrido del Río desde su nacimiento en la sierra de Albarracín hasta su desembocadura en Lisboa, llegando a la conclusión de que cuando el Tajo entra en Portugal llega casi "moribundo" y que la calidad de sus aguas sólo se recupera entre Abrantes y Lisboa. Esto porque el mayor Río de la Península Ibérica tiene que "soportar": primero, el trasvase hacia el Segura, que le merma en su capacidad de autodepuración; segundo los vertidos de 7 millones de madrileños - que según el reportaje, no están suficientemente bien depurados 6 ; tercero, los vertidos ilegales de mataderos, que tiran en el Río incluso esqueletos enteros de animales; cuarto, cuando llega a la Central Nuclear de Almaraz, las aguas del

${ }_{2}$ El día 24 de enero de 2019, el Río amaneció con un manto de espuma densa que cubría todo su cauce hasta Abrantes en lo que se puede considerar el culmen de más de dos años de contaminación sistemática del rio en la zona de "Vila Velha do Rodão" - reserva natural de la biosfera situada en la parte portuguesa del Parque Internacional del Tajo. El Ministerio del Ambiente portugués reaccionó inmediatamente procediendo a la limpieza de la espuma del Río que tanto impresionaba a las poblaciones vecinas y a la opinión pública en general a través de las imágenes en prime time de televisiones públicas y privadas, bien como de los restantes medios de comunicación. De facto, las espumas blancas fueron retiradas con relativa celeridad, sin embargo, no será tan fácil retirar los sedimentos del lecho del rio - contaminados por las mismas substancias. Según las declaraciones del Ministro de Ambiente português a la Agencia LUSA: "A operação de limpeza dos sedimentos no Tejo precisa de mais um mês para reunir todos os meios necessários e terá um custo entre 1 milhão e 1,2 milhões de euros..." Cf. LUSA, 21.02.2018: "Limpeza de sedimentos do Tejo exige meios específicos e custa até 1,2 ME - ministro".

3 Las licencias estaban atribuidas a la empresa de celulosa "Celtejo" y la APA ordenó el cierre de una empresa que se dedicaba al tratamiento de la aceituna "Centroliva", que simultáneamente fue acusada por la Fiscalía de crimen ambiental de contaminación de aguas por substancies consideradas muy nocivas para el ambiente y la salud pública.

4 Inclusivemente, la empresa Celtejo - una de las acusadas por el incidente de 24 de enero - alega que la gestión realizada por las Hidroeléctricas españolas aguas arriba impide que exista una buena calidad del agua ya que "secan" artificialmente el caudal del Río en determinados momentos, lo que disminuye su capacidad de carga, impidiendo la depuración natural del mismo.

5 Este reportaje fue portada en la Revista Sábado (de 15 a 21 de noviembre de 2018).

6 Lo que coincide con las últimas noticias sobre el río Manzanares, afluente del Jarama, que a su vez es afluente del Tajo: "La Fiscalía concluye que Getafe ha contaminado el río Manzanares durante 24 años La actual alcaldesa, del PSOE, está imputada por un delito contra el Medio Ambiente por estos vertidos" $(\mathrm{ABC}, 22 / 05 / 2019)$ 
Tajo se utilizan para enfriar los motores de la misma7, y; por último, los 120 embalses construidos del lado español, de los cuales, algunos de los de mayor dimensión, se encuentran en la provincia de Cáceres, justo antes de la frontera. Como es obvio, todos estos pantanos contribuyen para empeorar la calidad del caudal una vez que las "aguas paradas" propician fenómenos como la "eutropificación" o la "estratificación".

Durante 2019 los medios de comunicación portugueses han ido "bombardeando" la opinión pública con noticias alarmantes sobre el estado de las aguas del Tajo8 hasta que el 9 octubre noticiaban: "el Tajo internacional está en los mínimos de los últimos 40 años". El retrato desolador de los cauces secos del rio en territorio portugués se hace al por menor en reportajes televisivas alarmantes 9 , pero también en los restantes medios de comunicación10. En esta ocasión la "sequía" del Ponsul y Sever (afluentes del Tajo en territorio portugués) fue causada por descargas extraordinarias realizadas en la presa de Cedillo (situada en Cáceres junto a la frontera portuguesa y cuyo pantano es "alimentado" por los dos ríos antes referidos), con el objetivo de que España cumpliese in extremis el régimen de caudales anual de la Convención de Albufeira para el Tejo. A finales de octubre de 2019, varias voces provenientes de la sociedad civil11 afirman que la Convención de Albufera no fue cumplida en el Tajo, señalando como culpables en primer lugar los gobiernos, pero también las hidroeléctricas12. Otra vez, como en 2017, la historia se repite: el ministro del ambiente desmiente esa información,

7 Además en el reportaje se hace una mención a la noticia publicada por El País (03.10.2018) — “El Consejo de Seguridad admite la existencia de siete áreas en España contaminadas por radiación” — según la cual, en un afluente del Tajo, el Rio Jarama: "El Consejo de Seguridad Nuclear (CSN) ha admitido oficialmente - a través de un comunicado enviado a los medios la noche de este miércoles — la existencia de ocho banquetas con lodos contaminados radiológicamente en varios puntos de los márgenes del canal de riego del Jarama.

8 Ver, por dar otro ejemplo: “O maior rio da península ibérica está a morrer?" <http://expresso.sapo.pt/multimedia/2016-01-26-O-maior-rio-da-peninsula-iberica-esta-a-morrer->

9 Ver, por ejemplo, video de uno de los reportajes en TV: <http://195.23.58.155:8080/streamtv/2019/10/83013691.mp4>

10 Ver, por ejemplo: “Tejo. Ministério do Ambiente defende Espanha. Ambientalistas atacam” Diário I (09.10.2019).

11 Asociaciones de pescadores, clubes deportivos de navegación fluvial, alcaldes de los pueblos ribereños situados en el Parque Natural del Tajo Internacional e ONG de defensa del Ambiente. Cf: "Autarcas de Portugal e de Espanha preocupados com redução drástica do caudal do Tejo”, LUSA (07/10/2019)

12 “Quando as barragens secaram o Tejo!” - Cá por Causas” - por Paulo Constantino

<http://www.mediotejo.net/quando-as-barragens-secaram-o-tejo-ca-por-causas-por-paulo-constantino>. 
afirmando que España cumple los caudales13, y la CADC continua muda sin cumplir su papel de control de la aplicación de la Convención. Sobre los resultados del Grupo de Trabajo (GT) que fue creado el año pasado nada sabemos, una vez que el acta de la última reunión de la Comisión (realizada en noviembre del año pasado) todavía no está disponible en internet14 y este año no se reunió - aunque todavía está a tiempo de hacerlo para cumplir el mínimo exigido por la Convención (una vez por año). Todo parece indicar que la CADC no funciona con la diligencia que debía y los indicios del malestar del gobierno portugués en relación al Tajo manan de otras fuentes. Concretamente, en el nuevo Programa de Gobierno se prevé que el ejecutivo portugués inicie con el español un proceso de "desarrollo" de la Convención de Albufeira para garantizar caudales diarios que respondan a las necesidades ecológicas de los ríos internacionales y sus afluentes 15 y el pasado día 3 de noviembre el Ministro de Ambiente dijo públicamente que la gestión española de los caudales - aunque de facto cumpliese la Convención - era inaceptable ${ }_{16}$, debido a los daños económicos y ambientales causados.

\section{EL REFUERZO DE LA POLÍTICA DE COMBATE A LOS INCENDIOS Y ORDENACIÓN FORESTAL}

Como antes mencionamos, el actual "Ministério do Ambiente e da Ação Climática (MAAC)" adquirió nuevas competencias - antes atribuidas al Ministerio de Agricultura - y en lo que se refiere a la ordenación de los bosques.

Esta medida me parece fundamental para combatir con eficiencia los incendios forestales - recuerdo que los del verano/otoño de 2017 que provocaron 110 víctimas (amén de los costes ambientales y económicos). Una de las causas apuntadas fue precisamente la excesiva fragmentación de las competencias entre el Ministerio de Ambiente y el de Agricultura. Concretamente se refería la ineficacia de la tutela compartida sobre el "Instituto da Conservação da Biodiversidade e da Floresta" - que tradicionalmente estuvo siempre dirigido por

13 Tejo. Ministério do Ambiente defende Espanha. Ambientalistas atacam” Diário I de 09.10.2019.

14 Data de consulta 29/10/2019

$15 \operatorname{LUSA}(26 / 10 / 2019)$

16 «Governo: gestão que Espanha fez do caudal do Tejo “não é aceitável”». Público 03.11.2019. 
el Ministerio del Ambiente y que como consecuencia de la crisis económica de 2008-13 había pasado también a manos del Ministerio de Agricultura. La incompatibilidad entre los intereses ambientales y agrícolas ponían en causa la "gobernabilidad" y por consiguiente la eficacia en la actuación del referido Instituto.

El actual Gobierno refiere en su Programa que realizará una profunda reforma forestal, teniendo como meta una mayor resiliencia mediante el aumento del área de bosques y especies más adaptadas al territorio. Además, serán creados nuevos incentivos económicos para proyectos de sumideros forestales y otras actividades silvícolas y agroforestales que promuevan e secuestro de carbono. Este efecto también se conseguirá mediante la promoción de especies de crecimiento lento, de modo a asegurar la acumulación duradera de carbono atmosférico. No obstante, se estudiará la introducción de especies forestales no autóctonas más adaptadas a las nuevas condiciones climáticas de las regiones del país expuestas a la desertificación física y se clarificará el marco de responsabilidades en lo que toca a la ejecución de las redes de defensa forestal contra incendios. En las zonas afectadas por incendios de grandes dimensiones serán constituidas unidades de gestión que no solo atribuirán los incentivos financieros, sino que también aplicarán las multas a los propietarios o gestores de predios urbanos y mistos no edificados en el interfaz entre lo urbano consolidado y los espacios rurales.

Por último, hay que referir que en relación con las áreas protegidas y con el objetivo, entre otros, de prevenir los incendios en las mismas, fue aprobar el "Decreto-Lei" n. ${ }^{\circ} 116 / 2019$, de 21 de agosto por el que se establece el modelo de cogestión de áreas protegidas. Este modelo estaba ya previsto en la Resolución del Consejo de Ministros n.. 55/2018, de 7 de mayo, que aprobó la Estrategia Nacional de Conservación de la Naturaleza y Biodiversidad 2030 con la finalidad de establecer acuerdos de gestión colaborativa con las entidades presentes en el territorio de las referidas áreas protegidas. En este sentido, se tuvo en cuenta, la experiencia previamente adquirida con los proyectos de prevención estructural contra incendios y restauración de parques naturales y, en especial, el proyecto piloto para la gestión colaborativa del Parque Natural (PN) del Tajo Internacional, que tuvo su inicio en 2017, y que constituyó un 
modelo a seguir. En este caso, el acuerdo de cogestión reunió a la Autoridad Nacional para la Conservación de la Naturaleza y de la Biodiversidad juntamente con los tres municipios portugueses cuyos términos municipales están incluidos en el área del PN del Tajo Internacional, así como una institución universitaria, una asociación empresarial y una ONG de medio ambiente. Todas ellas intervinientes en el territorio portugués del referido PN.

Además, con el nuevo modelo de cogestión de áreas protegidas de ámbito nacional se permite que las asociaciones de municipios ya constituidas, o que vayan a constituirse, puedan participar en la gestión de áreas protegidas de ámbito nacional, siendo reconocidas como grupos de acción locales con capacidad para gestionar fondos europeos para el desarrollo local de base comunitaria. Así, se persigue la finalidad de imprimir una dinámica de gestión de proximidad en las áreas protegidas, bien como aplicar los principios de subsidiariedad, descentralización administrativa y participación de los órganos municipales en la respectiva gestión.

\section{EL ENORME AVANZO DE LA MOVILIDAD ELÉCTRICA EMPIEZA A DAR CLAROS FRUTOS}

Tal como en el año pasado, en 2018 continúa la tendencia de crecimiento del mercado de vehículos ligeros eléctricos, bien como la instalación de nuevos puntos de abastecimientos normales y rápidos que ya ha superado los 1350 , con los que se dio por concluida la $2^{-}$fase a finales de 2018 , extendiendo la red piloto de abastecimiento de vehículos eléctricos de acceso público a todo el territorio nacional. Así mismo, se ha dado continuación a los incentivos financieros y otras medidas de discriminación positiva para facilitar la utilización de vehículos eléctricos, pero además el nuevo gobierno pretende la eliminación de los subsidios prejudiciales al medio ambiente aplicados a los combustibles fósiles. Con esta finalidad, se procederá a una revisión de la fiscalidad sobre los vehículos para reforzar la discriminación positiva de los que tienen mejor desempeño ambiental, manteniendo la tributación en función de las emisiones de $\mathrm{CO}_{2}$ y confiriendo una clara ventaja fiscal a los vehículos eléctricos. Paralelamente se pretende alterar el marco fiscal, de modo a que as entidades patronales no faciliten coches de las empresas a modo de regalía salarial para 
sus empleados (o, por lo menos, en tal caso, sólo lo hagan con coches eléctricos), concediendo, en contrapartida, más ventajas para el copago mensual de transportes públicos para los trabajadores que opten por los mismos.

Para facilitar la transición, se prevé la adopción de un umbral de obligatoriedad de instalación de puestos de cargamento de vehículos eléctricos en determinadas infraestructuras de acceso público, como el interfaz de transportes municipales, bien como facilitar la instalación de puntos de cargamento domésticos y establecer la obligación de que todos los edificios nuevos dispongan de los mismos en sus garajes. Por su parte, y en lo que se refiere a bicicletas eléctricas, la meta fijada es alcanzar en 2030 la media europea. Actualmente, solo $0,5 \%$ de los portugueses usan la bicicleta como medio de transporte, por lo que el objetivo es llegar a los 7,5\%, lo que corresponde a un aumento de 15 veces más. Así, los dos mil quilómetros de vías para ciclistas existentes deben aumentar diez mil hasta 2030 y se avanzará en medidas de educación para aumentar la seguridad vial y la compatibilidad entre los diferentes tipos de vehículos que circulan por la vía pública, a fin de reducir a mitad la siniestralidad de peatones y ciclistas. En este campo, de acuerdo con la Federación Europea de Ciclismo, Portugal ocupa a 27.a posición, entre los 28 países con "condiciones para pedalear".

Por último, en los que se refiere a los patinetes eléctricos, la policía municipal de Lisboa ya incautó más de 2200 en lo que va de año y las multas por patinetes eléctricos mal estacionadas superan los 17 mil euros.

\section{PARTICIPACIÓN PÚBLICA}

\subsection{Novo aeropuerto de Lisboa}

Continua durante este segundo semestre de 2019 la fuerte polémica en torno del proyecto de construir un segundo aeropuerto para Lisboa que se situaría en una antigua base aérea militar - la Base Aérea n. 6 , localizada en el distrito de Setúbal, entre Montijo y Alcochete (municipios situados justo en frente de Lisboa, pero del lado sur del Estuario del Tajo en la salida del Puente Vasco de Gama).

La necesidad de ampliar el aeropuerto de Lisboa surge porque el actual conocido como "Aeroporto da Portela" - se encuentra muchas veces colapsado 
por la afluencia masiva de turistas desde 2013 aproximadamente. Antes de la crisis económica de la zona euro, existía un proyecto para construir el mismo en la Base Militar de Alcochete. Pero este proyecto, que fue sujeto a Evaluación de Impacto Ambiental (EIA) y tuvo una declaración positiva fue, sin embargo, abandonado, por ser demasiado oneroso - esta decisión fue adoptada en el momento en que el Estado portugués tuvo que ser rescatado por la denominada "troika" (FMI, BM e CE).

Aunque, inicialmente, el actual gobierno refirió que no sería necesaria la realización de EIA - ya que las dimensiones de Montijo eran muy inferiores a las de Alcochete -, la verdad es que el proceso está en marcha. El pasado día 19 de septiembre concluyó la fase de consulta pública, con 928 participaciones registradas en el portal Participa - gestionado por la "Agência Portuguesa del Ambiente" (APA). El 10 de octubre, el presidente de la APA anunció en el Parlamento que ya tenía toda la información necesaria para concluir el EIA por lo que la declaración de impacto ambiental (DIA) seria conocida a finales de octubre.

Aunque siendo de menor envergadura que el proyecto de Alcochete, la inversión seria superior a 1,15 mil millones de euros, lo que incluye también la construcción de un nuevo acceso ferroviario, que permitirá establecer la conexión del Aeropuerto do Montijo con la autopista A12 y la Terminal Fluvial del Seixalinho y también la construcción de una pista para bicicletas a lo largo de este acceso.

Tanto las instituciones (municipios, asociaciones y partidos políticos) como los particulares están fuertemente divididos. Casi todas las ONG y asociaciones de medio ambiente son contra un proyecto que significa aumentar dramáticamente la mortandad de las numerosas aves que nidifican y tienen en sus rutas la travesía del Estuario del Tajo. Rutas que podrán colisionar con los vuelos de las aeronaves, causando graves accidentes. Además, toda la legislación Internacional de la UE e, incluso, nacional condiciona y limita este tipo de actividades en zonas especialmente protegidas, como lo es el Estuario del Tajo. En este sentido, se puede citar, por ejemplo, la Convención Ransar, la Directivas Hábitats y la Directiva Aves, bien como la legislación portuguesa sobre reservas naturales. Pero, además, las ONG piensan que la firma prematura del Acuerdo entre el gobierno y la ANA (Aeropuertos de Portugal, liderada por la francesa Da 
Vinci) para la construcción de la obra de Montijo, antes de la elaboración del do Estudio de Impacto Ambiental (EIA) y del parecer de la Comisión de Evaluación es una forma de intromisión política en el proceso de EIA.

Entre los municipios, las opiniones divergen de acuerdo con la familia política a que pertenecen alcaldías. En cuanto que los ayuntamientos del partido socialista (PS), piensan que el aeropuerto es una oportunidad para el desarrollo económico sostenible - es el caso de Barreiro y Montijo - el Municipio de Moita y Seixal (cuyas alcaldías pertenecen Partido Comunista Portugués (PCP) defiende justamente lo contrario. O sea, que el nuevo aeropuerto, además de los impactos ambientales ya referidos por las ONG, perjudicará la calidad de vida de sus poblaciones, debido al ruido y aumento del tráfico y del valor de la vivienda. Argumentan, también, que la opción actual es de corto plazo, pues el turismo en Lisboa está creciendo de tal manera que rápidamente el nuevo aeropuerto será insuficiente y habrá que construir otro de dimensiones superiores, duplicando el gasto público - en este sentido la opción inicial (proyecto de Alcochete) era más ventajosa para el interés general.

Por otra parte, los especialistas en protección civil, consideran el local escogido poco seguro para hacer un aeropuerto, debido a los riesgos de proximidad de un depósito de 175 mil metros cúbicos de combustibles y productos químicos, a los riesgos sísmicos de la zona y a las dificultades de socorro en caso de accidente en pistas de aterrizaje que terminan en zonas del Estuario de Tejo cubiertas de lodos.

Finalmente, el "Instituto de Conservação da Natureza e das Florestas" emitió un parecer favorable al EIA, pero condicionado a que la "ANA Aeroportos" pague cerca de 20 millones de euros para mitigar y compensar los impactos en la avifauna. A razón de $€ 4,5$ por cada movimiento (aterrizar y despegar) en el nuevo aeropuerto. Por último, el actual Gobierno defiende la localización del nuevo aeropuerto en Montijo, argumentando que será mucho menos oneroso para el erario público, de menor dimensión que Alcochete pero mucho más próximo de Lisboa.

Justo cuando estaba concluyendo esta crónica, el pasado 31 de octubre fue publicada la decisión (DIA) favorable de la APA para ejecución del proyecto. $O$ 
sea, todo indica que Lisboa va a tener nuevo aeropuerto en Alcochete, si bien que las medidas de compensación para minimizar los impactos del mismo ascenderán a $€ 48$ millones. No obstante, hay que referir que está todavía pendiente la decisión sobre proceso judicial iniciado en febrero por una ONG de defensa del medio ambiente que demanda la realización de una evaluación ambiental estratégica (EAE) - que, en su opinión, debería haber sido realizada antes del EIA del proyecto - y que ya comunicó públicamente que está ponderando interponer una providencia cautelar sobre la DIA que aprueba el nuevo aeropuerto17.

\subsection{Programa Nacional de la Política de Ordenación del Territorio (PNPOT)}

El pasado 5 de septiembre fue publicada la Ley $n .0$ 99/2019, que realiza la primera alteración al PNPOT que se había aprobado mediantes la Ley $\mathrm{n} . \stackrel{0}{0}$ $58 / 2007$ con la finalidad de adaptarlo a los nuevos desafíos, como por ejemplo las directrices de la Estrategia de Ciudades Sostenibles 2020 o los compromisos adquiridos con base en el Acuerdo de París sobre el cambio climático. La referida ley fue precedida da correspondiente fase de consulta pública que contó con una amplia participación, una vez que el PNPOT es el instrumento principal del sistema de gestión territorial, define los objetivos y opciones estratégicas de desarrollo territorial y establece el modelo de organización del territorio nacional.

La figura del PNPOT fue creada por la Ley de Bases de la Política de Ordenación del Territorio y de Urbanismo de 1998, con el objetivo de dotar al País de un instrumento necesario para obtener una visión prospectiva, completa e integrada de la organización y desarrollo del territorio y para promover da coordinación y articulación de políticas públicas con base territorial, bien como para la programación de inversiones territoriales a financiar tanto por programas nacionales, como por los de la Unión Europea (UE). Los elementos fundamentales del PNPOT son: por un lado, el Modelo Territorial que sintetiza los principales sistemas territoriales, y; por otro lado, la Agenda para el Territorio que contempla 50 medidas políticas para dar respuesta y aprovechar positivamente los cambios radicales que el país va a sufrir, así como apoyar los 
nuevos cambios de rumbo que se pretenden concretizar para alcanzar el desarrollo territorial sostenible.

Entre las medidas más importantes que se pretenden adoptar en relación con la Política de Medio Ambiente con incidencia en el territorio, están: mejorar la gestión del recurso agua en un clima de escasez; valorizar el recurso suelo y evitar el desperdicio del mismo; afirmar la biodiversidad como un activo territorial; valorizar el territorio a través del paisaje; planificar y gestionar de forma integrada los recursos geológicos y mineros; ordenar y revitalizar los bosques; prevenir riesgos y adaptar el territorio al cambio climático; valorizar el Litoral y aumentar su resiliencia, y; promover la rehabilitación urbana, cualificar el ambiente urbano y el espacio público.

\section{OTRAS NOVEDADES DE INTERÉS: NEUTRALIDAD CARBÓNICA, ECONOMIA CIRCULAR Y LISBOA CAPITAL VERDE 2020}

\subsection{Neutralidad carbónica}

En materia de combate al cambio climático, fue aprobada en julio la Resolución del Consejo de Ministros" n. ${ }^{\circ}$ 107/2019, por la que se aprueba la "Hoja de Ruta para la Neutralidad Carbónica”. De acuerdo con la misma, para que Portugal sea neutro en carbono en 2050 habrá que reducir, a partir de 2019, de 68 para 12 Megatoneladas las emisiones de $\mathrm{CO}_{2}$. Lo que significará aumentar de 9 para 12 Megatoneladas la capacidad de secuestro forestal del país. En 2030, el 80\% de la energía eléctrica producida en el país deberá ser producida por fuentes renovables y en 2050 deberá ser 100\%. Así, la dependencia energética del exterior, que es hoy de 75\%, en 2030 será de 65\% y en 2050 de 17\%. El uso de petróleo que hoy ultrapasa los 65 millones de barriles/año, no deberá de superar los 10 millones en 2050 y dejará de ser usado en la movilidad terrestre. El ahorro anual derivado de las importaciones de combustibles - que dejarán de ser necesarias - alcanzará los 4 mil millones de euros por año, lo que representa un valor acumulado (entre 2020 e 2050) de 128 mil millones de euros.

En lo que se refiere a la inversión estatal necesaria para las medidas previstas en la referida Hoja de Ruta se calcula que en media anual - fuera otros gastos necesarios e indirectos -, serán aplicados directamente: en el ciclo urbano del 
agua, alrededor de 500 millones de euros; en la adaptación al territorio - litoral y combate a las inundaciones y sequías - aproximadamente 200 millones, y; para la "descarbonización" de los transportes y eficiencia energética irán hasta los 1200 millones. Pero también se espera que la dinámica económica del mercado, apoyada por un conjunto de políticas públicas adecuadas - sobre todo las relacionadas con el Plan para la Economía Circular -, originen una inversión privada anual de 30 mil millones de euros entre 2020 e 2050.Como balance final, la Hoja de Ruta concluye que: sin el impulso de la neutralidad carbónica habría una reducción de $60 \%$ de las emisiones de $\mathrm{CO}_{2}$ hasta 2050, mientras que, gracias a ella, se espera alcanzar, por lo menos, $85 \%$.

Hay que referir que una das medidas más polémicas de la Hoja de Ruta fue la previsión de una reducción significativa del número de ganado bovinos, debido a su fuerte contribución para las emisiones de metano. Para apoyar esta medida, el Rector de la Universidad de Coimbra decidió eliminar la carne de vaca del menú de los comedores universitarios a partir de enero de 202018. Está decisión fue objeto de alguna discusión en los medios de comunicación, que la han valorado tan sólo como una medida simbólica con pocos efectos prácticos, pero que ha levantado la "furia" del sector ganadero.

Por último, el programa de gobierno, para apoyar las medidas de la Hoja Ruta para la neutralidad carbónica prevé también el desarrollo de un "banco verde". El objetivo es desarrollar, a través de la "Institución Financiera de Desarrollo", un banco verde para conferir capacidad financiera y acelerar las varias fuentes de financiamiento existentes dedicadas a proyectos de neutralidad carbónica y de economía circular.

\subsection{Economia circular}

En esta materia, el nuevo gobierno pretende aplicar medidas de fiscalidad verde, para conseguir un progresivo reequilibrio de la carga fiscal, disminuyendo los impuestos sobre el trabajo y penalizando las prácticas que perjudiquen el medio ambiente, como el consumo excesivo y la no reutilización y reciclaje de residuos. 
Sin embargo, los datos sobre los resultados de una de las medidas más simbólicas de fiscalidad verde19" - la destinada a reducir el consumo de bolsas de plástico - continúan siendo poco prometedores. Con efecto, muestran que los portugueses están a reducir progresivamente su consumo, pero quien se está beneficiando son los hipermercados y no la Hacienda Pública. Así, el Ministerio de Ambiente informó que la recaudación fiscal realizada en el primer año de aplicación de la tasa fue de tan sólo $0,4 \%$ de la inicialmente prevista, por lo que el Estado arrecadó cerca de dos mil euros el año pasado. Estos datos fueron publicados recientemente por el Instituto Nacional de Estadísticas (INE) y muestran, sin embargo, que en 2018 los ingresos con impuestos ambientales subieron en su totalidad para 5,3 mil millones de euros.

Así, en lo que se refiere al capítulo de los plásticos el gobierno pretende avanzar con una nueva medida: abolir, hasta finales de 2020, los plásticos no reutilizables (como platos, vasos, cubiertos de plástico o pajitas), anticipando en un año la aplicación de la directiva europea sobre la materia y definiendo un horizonte próximo, pero realista, para a abolir progresivamente otros usos de plástico, como los excesos en embalajes de productos, a través de sistemas de depósito, de mecanismos de reutilización y de ecodesign que permitan minimizar los residuos. También se pretende estimular a las empresas a que asuman compromisos voluntarios de eliminación o reducción de plástico en los embalajes de sus productos, especialmente en el sector alimentar y en las entregas a domicilio. Además, fue aprobado una norma que será fundamental para comenzar a reutilizar el agua: el "Decreto-Lei" n. ${ }^{\circ}$ 119/2019, de 21 de agosto, que establece el régimen jurídico de producción de agua, obtenida a partir del tratamiento de aguas residuales, para reutilización.

\subsection{Lisboa capital verde 2020}

Por último y para acabar con una noticia esperanzadora, hay que referir que Lisboa fue galardonada con el premio "Capital Verde Europea 2020". La distinción fue anunciada por el Comisario Europeo do Ambiente, Asuntos Marítimos y Pescas, constituyendo un reconocimiento al trabajo que Lisboa ha 
desarrollado durante la última década para mejorar el ambiente urbano y la calidad de vida de los lisboetas. Es la primera vez que una capital del Sur de Europa conquista esta distinción, generalmente atribuida a las ciudades del Norte - la próxima es Oslo. Pero, además la "ambición ambiental” de Lisboa no acaba aquí, sino que también se sumó al C40, Grupo de Lideranza Climática de las Grandes Ciudades. Esta red tiene como objetivo el combate al cambio climático a escala de las grandes metrópolis - entre la que se cuentan 94 de las mayores del mundo - como Paris, Londres, Los Ángeles, Boston, Milán, Tokio o Seúl -, representando más de 700 millones de habitantes y un cuarto de la economía mundial.

El jurado que escogió Lisboa como Capital Verde destaco el hecho de haber sido la primera capital europea a firmar el nuevo Pacto Global de los alcaldes para el Clima y la Energía, bien como haber reducido substancialmente las emisiones de $\mathrm{CO}_{2}$, el consumo de energía y agua 20 y tener una visión clara para la movilidad urbana sostenible. Sin embargo, el turismo no puede quedar al margen de la descarbonización y en Lisboa ha aumentado considerablemente21. En este sentido algunos 22 barajan la posibilidad de convertir la denominada "tasa turística" - que en este momento es de 2 euros por turista y por noche - en una tasa local para la descarbonización que cobre al turista un valor correspondiente a su huella carbónica y que se destine la mitigación de la misma. Medir el impacto ambiental turístico y concretizar su valor puede ser el primer paso para reducir su huella carbónica sin dejar de promover esta lucrativa actividad económica. Pero como toda actividad, la misma debe tener algún límite antes de pasar para el lado de la insostenibilidad ambiental. De ahí la gran polémica alrededor del nuevo aeropuerto de Lisboa, recientemente aprobado, que además de los impactos ambientales de un proyecto de esta envergadura que será construido

20 La Cámara de Lisboa prevé el ahorro de cerca de 75\% del agua potable hasta 2025, a través de un plan de reutilización de agua con una inversión de 16 millones destinado a crear una nueva red de agua reciclada para consumo no potable. Esta nueva red se servirá del agua reciclada en las tres EDAR de Lisboa e incluye la construcción de $55 \mathrm{~km}$ de conductas aductoras principales, 16 nuevos sistemas de elevación y 12 reservatorios.

21 De acuerdo con los datos del Instituto Nacional de Estadísticas (INE) 58\% dos 67 millones de dormidas de turistas registradas en 2018 en Portugal se realizan en 3 ciudades: Lisboa (13 millones), Oporto (4 millones), Cascais (1,5 millones).

22 En este sentido ver la opinión de FELIZARDO, José Rui, y FERREIRA, Lurdes en “Descarbonizar as cidades sim, mas com o turismo. As cidades precisam de uma taxa local para a descarbonização que as ajude no papel cada vez mais central de reduzir as emissões de $\mathrm{CO}_{2}$ do planeta”. Público, 31/10/2019. 
junto al Estuario del Tajo, servirá para aumentar el número de vuelos low cost y el número de turistas de una Lisboa que comienza a dar "señales de cansancio". A modo de conclusión, puedo decir que, en mi opinión, Lisboa está como una rosa, pero no hay rosa sin espina. 